\title{
Bio-Chemical Sensitivity of Organic Semiconductors for Smart Electronic Sensors
}

\section{Hadayat Ullah Khan*}

Materials Science and Engineering Program, Division of Physical Science and Engineering, King Abdullah University of Science and Technology, Thuwal 23955-6900, Saudi Arabia

We are pleased to publish the 1st issue of Organic Electronics and Biosensors, in an international Journal of Biochips \& Tissue Chips that is intended to provide top-quality peer-reviewed articles in the fascinating field of bioengineering particularly in the area of structure, synthesis and processing, characterization, advanced-state properties, applications in biochips and biosensors.

With the struggle of chemists and chemical engineers, fused-ring compounds with the integration of sulphur atoms in the center of the molecules and addition of side chains not only increased the ambient stability, but also enhanced the mobility with improved microstructural geometry, necessary for an efficient transfer of charge from moleculeto-molecule to conduct electricity in field-effect transistors (FETs). FETs are one of the fundamental building blocks in emerging organic electronics, in which the conductivity of a semiconducting layer measured by two metallic contacts can be drastically modulated by a voltage appliedto a third electrode, called the gate.

Such simplifying science is essential particularly for the development of emerging areas such as plastic electronics, bionics, synthetic biology, energy harvesting and biosensors. This journal will facilitate the open exchange of techniques and methodologies designed to bridge traditional disciplines and to connect the energies of scientists world-wide in delivering novel materials for a new generationof devices including bio -chemical sensors to molecular electronics. The combination of such diagnosis with pharmaceutical not only provides immense clinical benefit but provides a new financial model to drive forward the progress of new electrical sensors. Although biosensors have been around since glucose monitors were commercialized in the 1962s, the transition of laboratory research and numerous research papers on biosensors into the world of trade has trailed.Regarding the huge sector of medical diagnostics (with a market volume of $\$ 30$ billion), the state of the art dictates that a trustworthy patient profile requires a very large number of test, each one dedicated to the detection of an individual target analyte. This is by no means common in the case of complex aliments, such as genetic disease. Having this in mind, scholars have motivated their strength both the improvement of disposable DNA biosensors as well as on creating systems able to provide a selective, sensitive and at fast speed with low cost.

DNA hybridization involves the bonding of a single strand of DNA (from the solution) to another known single-stranded DNA molecule (covalently attached to the transducer surface). Hybridization can occur only if the target and the probe have a complementary sequence of nucleotides, enabling them to double strand. As a result, when the target DNA is delivered to the device, stable hybridization took place and changed the conductivityof the transistor. This electrical response systematically depended on the complementarity (the degree of matching) of the targeted DNA and the probe, and creates possibilities for the low-cost, in-situ electronic detection of DNA without the use of radioactive of phosphorescent labels and expensive conventional DNA detection systems.

Thus, we see a vision of the future including the manipulation of nanotechnology, smart materials and creation of plastics bioelectronics. The forthcoming may not be clear but evolving new prototypes in biosensors and bioelelectronics assure to furnish an exciting interdisciplinary environment rich in novelty to befit medical society.

*Corresponding author: HadayatUllah Khan, Materials Science and Engineering Program, Division of Physical Science and Engineering, King Abdullah University of Science and Technology, Thuwal 23955-6900, Saudi Arabia, E-mail: Hadayat.Khan@kaust.edu.sa

Received June 22, 2012; Accepted June 25, 2012; Published June 28, 2012

Citation: Khan HU (2012) Bio-Chemical Sensitivity of Organic Semiconductors for Smart Electronic Sensors. J Biochips Tiss Chips S8:e001. doi: 10.4172/2153-0777. S8-e001

Copyright: (c) 2012 Khan HU . This is an open-access article distributed under the terms of the Creative Commons Attribution License, which permits unrestricted use, distribution, and reproduction in any medium, provided the original author and source are credited. 